\title{
$\nabla$ Segmentation of Features Using Neural Network with Cardiac Dataset
}

\author{
IJCRR \\ Section: Healthcare \\ ISI Impact Factor \\ (2019-20): 1.628 \\ IC Value (2019): 90.81 \\ SJIF (2020) $=7.893$

\section{Ramamoorthy V'1, Deepa $D^{2}$, Cherukullapurath Mana $\mathbf{S}^{3}$, Sivasangari A4, Samhitha BK ${ }^{5}$, Judgi T}

${ }_{1,2,3,4,5,6}$ School of Computing, Sathyabama lnstitute of Science and Technology, Chennai, Tamilnadu-6oon19, India.

\section{ABSTRACT}

Introduction: One of the most popular applications of Artificial Intelligence that has seen immense growth in the digital era is Deep Learning techniques where the system studies and improves its performance through progressive learning without any explicit programming. Deep learning is widely used in numerous applications and one of them being medical analysis. Feature extraction and Image classification are considered to be the most popularly used approaches done using the deep learning process.

Method: In this paper, we will segment cardiac bi-ventricle from magnetic resonance (MR) images. The segmented images are then classified through Deep Neural Networks where the sequence of images is validated frame by frame. The efficiency of the proposed model is evaluated and is compared with other traditional Deep Learning processes.

Result: Theexecutionof the model is more precise as the model uses an iterative approach for feature extraction in classifying images.

Conclusion: It is observed that the proposed interactive model provides better performance.

Key Words: Medical analysis, Classification, Deep learning, Segmentation, Artificial neural network, Feature extraction

\section{INTRODUCTION}

The development of Artificial Intelligence has provided birth to a lot of brand-new solutions, from that the favourite solutions which are being developed currently are Machine Learning Approaches as well as Deep Learning Techniques. These mastering methods are commonly used within several fields such for instance healthcare picture evaluation ${ }^{1}$ automatic robot course preparing ${ }^{2}$ flood detection within a specific area or city ${ }^{3}$ and also acreage coverage category. ${ }^{4}$ Printer mastering strategy is a procedure for studying a certain undertaking with no man treatment as well as enhancing the overall performance just by constant mastering procedure. The mastering methods are of two types: supervised learning, ${ }^{5}$ where the category classification is designed for the functions on the instruction dataset as well as unsupervised studying ${ }^{6,7,8}$ exactly where absolutely no product labels are provided and also other method has to tag the characteristics on the dataset. This Include removal is a crucial practise within the entire printer mastering techniques. ${ }^{9}$ The extracted capabilities might subsequently be utilized for different methods as regression or classification. The utilization of ANNs is largely known as Deep Learning Approach. When the community striving to learn every single level quite profoundly requires the result of every level because they form the centre of upcoming coating methods. ${ }^{10,11}$ ANNs can serve as a classifier similar to the performance associated with a natural neuron Owning many levels attached by way of weights. ${ }^{12}$

Within picture processing, the quantity of pixels (picture elements) is dependent on the type of picture. The impression is the imitation of truth that provides just as much info as you can approximately an item. The setup of neurons being created levels and also the hookup patterns created within as well as in between every level is known as Network Structures. ANNs have information processing components that could fix any kind of difficulty via pre-determined examples instead of pre-defined algorithms. ${ }^{13}$

\section{Corresponding Author:}

R. Vignesh, Department of Computer Science and Engineering, Sathyabama Institute of Science and Technology, Chennai 600119, India. Phone: 9597370245; Email: vignesh.star975@gmail.com

ISSN: 2231-2196 (Print)

Received: 05.12 .2020
ISSN: 0975-5241 (Online)

Revised: 27.01 .2021
Accepted: 08.03 .2021
Published: 20.07 .2021 
Deep Learning Approaches discovers a distinguishing value in the area of healthcare reputation evaluation. Pictures about the measurement of different areas of the body based upon various scales such as macroscopic or microscopic are coined as biomedical photographs. These biomedical pictures are produced although numerous tools including U1trasound devices as well as CT scanners. With this approach, we suggest a method of determining as well as segmenting cardiac bi ventricle offered by MR pictures. The suggested method applications of Region of interest (ROI) Algorithm which recursively processes the pictures within the data source to determine the malignant cells as well as sector it out of various other areas of the body. Other parts of the image are made of a few similar segments which are effective and recommended by numerous scientists, the methodologies \& algorithms employed within the suggested strategy as well as experimental outcomes.

\section{RELATED WORK}

A lot of effective studies are suggested by different scientists for segmenting or maybe classifying a certain item or even a particular location coming from a picture. A semi instant segmentation technique suggested. Believed the number of tumour cells located inside the liver. ${ }^{14}$ The borders of the tumour cells have been pinpointed by a CT image. This task undertaken can use so much computation power and time. It is advertised to be effective in the process of locating the number of tumour cells present by segmenting them into segments. When it comes to hybrid picture segmentation, it is utilizing morphological algorithm for watersheds which consolidated advantage \& area-based methods. ${ }^{15}$ The method discovered to become effective because it decreased the quantity of false-positive detection. Radiation of sound aided to the aspect for tacitly enhancing the processing phase of computation. ${ }^{16}$ Created an effective and also strong supervised reputation co segmentation design containing a method known as style incentive as well as an energetic contour version. The unit was examined on several pictures coming from a data source as well as may effectively match the typical items with little mistake fee.

Brain tumour as detected by Jason J. Corso in ${ }^{17}$ by integrating segmentation with Bayesian model. A weighted aggregation algorithm was used for the detection of tumour cells. The performance evaluation was done on a larger dataset where stochastic models were used to extract the features. The model could be enhanced by providing an accurate boundary of the tumour cells. Concurrent image segmentation and bias adjustment were performed by Kaihua Zhang, ${ }_{18}$ where minimization of energy was performed by an efficient Bayesian Learning Approach. The technique stated that the experimental results were performed on a real dataset of images and produced an accurate intensity of homogeneity. Md.
Badrul et al. ${ }^{19}$ classified lung cancer images from CT scans using the MLFFNN technique yielding a good accuracy of $96.67 \%$. FFNN approach used $i^{20}$ describedtheclassificationtechniqueproducingan accuracy of $92 \%$. Rajesh Kumar Tripathy ${ }^{21}$ combinedSVMwithLeastSquareandprovidedan accuracy of $95.34 \%$. Persi et. al. $2013^{22}$ used

The particle swarm optimization technique for predicting heart disease yielding $92.2 \%$ accuracy. Deepa et.al. ${ }^{23}$ proposed an idea of detecting road damage by image processing in smartphone and sending the co-ordinate point to the cloud from cloud user can visualize the road where the damages there it will show in the map. From this can able to avoid the accidents etc.

Similarly Keerthi et.al ${ }^{24}$ talks about CNN to identify tumors that are dangerous in lung disease. The $\mathrm{CNN}$ technique has the lot features and standard representation pneumonic radiological complexity, fluctuation and classification of a lung nodule. Similarly, Vignesh et.al ${ }^{25}$ discussed fewer deployment in the cloud storage with low-cost replication, higher availability with better performance in geo-replicated systems by data centres with these benefits.

Further Ishwarya et.al ${ }^{26}$ proposed a project to reduce congestion in traffic and calculating current traffic with normal. If any unusual them emergency passes through the signals. Based on that solves the traffic problems.

\section{MATERIALS AND METHODS}

Numerous feature extraction methods and classification techniques are used widely to identify and detect the location of malignant cells such as tumour cells in the human body. Various classifiers such as SVM, K-means clustering and decision trees that are used widely for image segmentation applications have been already discussed. The enhanced and efficient model designed in the current paper first extracts images from an MR image dataset. The images are then pre-processed where all the features of the images are extracted using neural networks. The preprocessing is initially done by medical image analysis where all the images are processed in such a way that it generates new fused images that have high quality when compared to the original images. These fused images are easier for training and classifying the classifier as it contains more spatial and spectral information. The validation of the data set is done frame by frame for a particular period. As the proposed model is an iterative process, the model tends to produce a more accurate result when compared with other existing classifiers. The various steps involved in the entire model is discussed as follows:

Image Acquisition: The scanned images obtained from patients suffering from cardiac stroke are collected from data- 
bases and hospitals. The file formats collected from the database are.jpeg, tift, .png file formats and the file dimensions consist of rows and columns.

Image Pre-processing: The purpose of image-processing is to increase the quality of the image and to improve the features of an image for further processing. After the image acquisition stage, the images pass through the pre-processing stage. There will be a change in the output image for the given input image. The occurrence of this change is due to the reduction of noise and/or enhancement of contrast. Image pre-processing is needed to vary its lightning condition. Preprocessing entails Color Normalization, Edge Detection, Noise Reduction, as well Histogram Equalization. A lot of filter systems are accustomed to bringing down the outcome of interference over the picture. Intermediate Filter is utilized for Color Normalization and Noise Reduction. Gabor Filter has utilized for Edge Enhancement as well as Histogram Equalization. Picture Enhancement is used to enhance the notion of information of pictures. Additionally, it modifies the characteristics of a picture and also causes it to be ideal for a job. The highest Peak Signal to Noise Ratio (PSNR) is estimated for all the segments and various picture information is established. The established good signal-to-noise ratio (PSNR) is used with a phrase for relating the ratio in between the optimum probable worth (energy) of the power and a signal of distorting racket which affects the calibre of the representation of its.

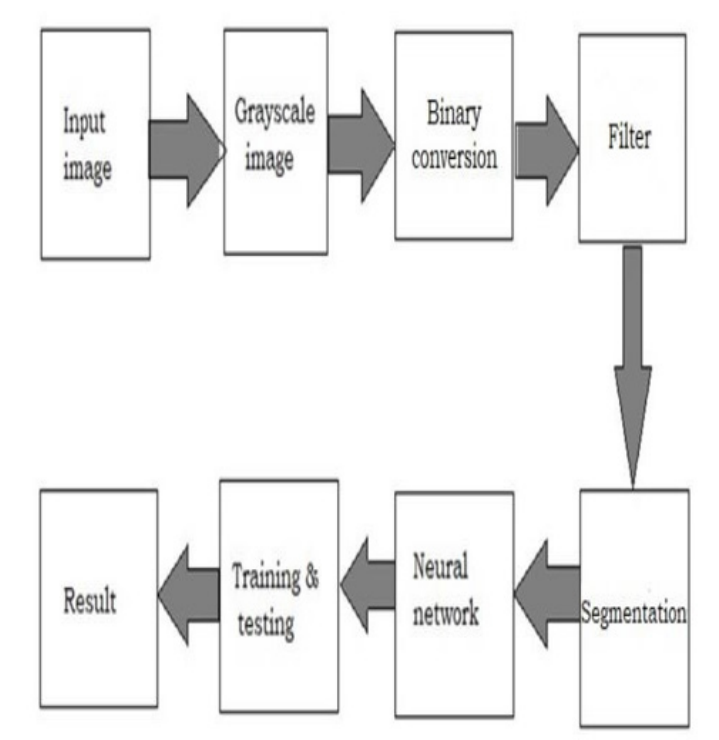

Figure 1: Workflow of Classification of images.

Feature Extraction: It is a method of capturing the visual content of the image. It is extracted from the segmented image which includes area, perimeter, equivalent diameter, irregularity index, mean, standard deviation and entropy. ExtractedFeatures are used in a neural classifier to train the model in this way to increase accuracy and recognize a par- ticular class as normal or abnormal. The classifier will assign the unknown object to the correct class depending on the extracted features (Fig. 1).

Segmentation of Malignant Cells: In this process, the homogeneous regions are obtained from the input image. The region so interesting images are found by using the process of segmentation. It reduces the number of pixels of an image to make it easier for the subsequent step of feature extraction and classification. Segmentation is tougher in MR scan images and when combined with a large amount of data because of the additional dimensions that need to be considered for the proximity calculation. Fig. 1 depicts the flow chart of the entire classification process.

In Fig. 2 we have depicted the entire architecture of how the classification is done. The Input image or a video frame is given from the scanner database. In the case of a video file from the scanner, it is split into multiple frames and then is first converted into greyscale. The discrete cosine transformation method is applied to the image when the contrast of the image is enhanced in such a way that even the small feature soft images are perfectly visible. Thenthefeaturesareextractedfor further study and then finally Medical Image Fusion is performed on the image to obtain the segmented image. Once the fused images are obtained it is then trained using neural networks which are responsible for extracting the features of the image. Then required performance and the error calculation is done for the entire model.

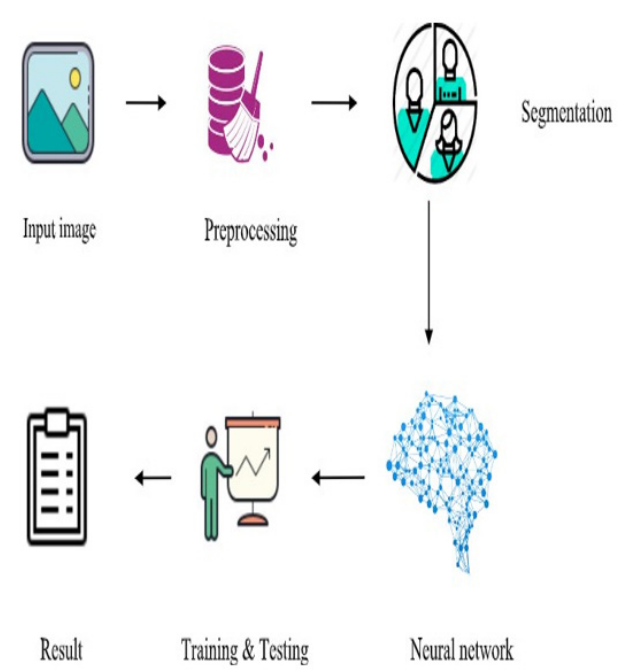

Figure 2: Architecture Diagram Proposed System.

\section{RESULTS AND DISCUSSION}

The experiments are completed on the MATLAB R2018a version. The calculations are performed using the image Classification Learner Toolbox(ICLT) that is readily available in MATLAB.Initialstepistoinputanimagefromthe scan- 
ner database into the given tool. Fig 3 is a sample output image obtained after the classification using the neural network classifier. The cardiac bi-ventricle is can be easily detected from this classification.
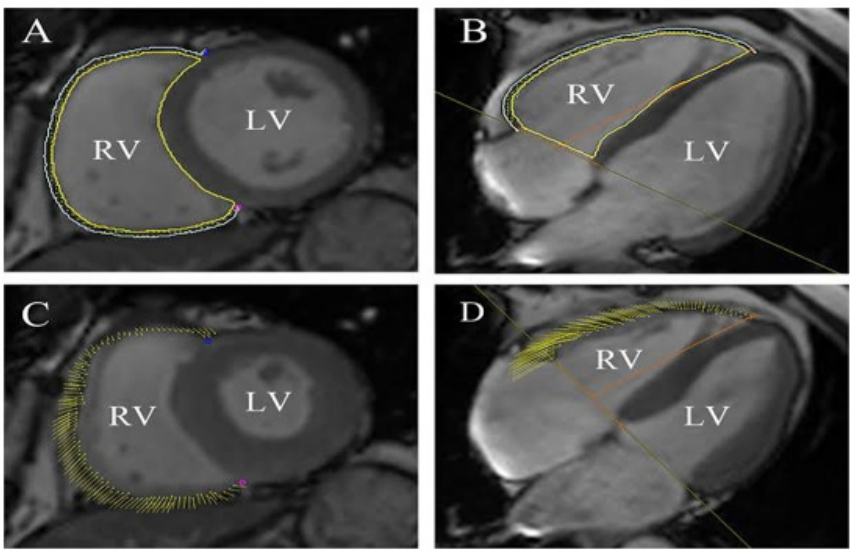

Figure 3: Classified Output.

As the next step, the RGB components are extracted from the image and then converted to a Grayscale image. The features are then trained with a neural networks classifier where some images are reserved for training and the rest of the images are used for testing of images. The classifier learns features from the given images and successfully classifies when any new image is given to it. It identifies where the image is benign or malignant. The accuracy of the neural network is observed with $99.69 \%$ whereas $99.9 \%$ specificity and $80.48 \%$ sensitivity. The segmentation of the cardiac bi-ventricle from the exact image gives us various parameters for measuring such as its intensity, volume, and size. This helps in diagnosing and treating cardiac-related issues more effectively. The results show the efficiency of the proposed approach.

\section{CONCLUSION}

Numerous cancerous cells and tumour cells are prevailing in a lot of people nowadays. Early detection of these malignant cells could reduce the risk of loss of life to a greater extent. In this paper, we have suggested an efficient model of diagnosing cardiac bi-ventricle at an earlier stage by using Medical Image Analysis. The images obtained from MRI scanners are used for fusion in such a way that generates high image quality. The images are classified using deep neural networks. The region of Interest (ROI) algorithm is used to segment the area affected. These images are then used for further classification. Neural networks are used for classification and have obtained an accuracy level of about $99.69 \%$. As future work, we are currently working on improving the proposed algorithms to give better efficiency and more accurate predictions.

\section{Conflict of Interest}

The authors declare that there is no conflict of interest involved

\section{ACKNOWLEDGEMENT}

We acknowledge the contributions made by peer researchers which motivate us to research this domain.

\section{Funding}

Not Applicable

\section{Author Contribution}

1. R. Vignesh - Data Analysis

2. D. Deepa - Manuscript planning

3. Suja CherukullapurathMana- Data Collection

4. A. Sivasangari- Implementation

5. B. KeerthiSamhitha - Analysis of Result

6. T. Judgi- Manuscript Planning

\section{REFERENCES}

1. Rahman MM, Bhattacharya P, Desai BC. A framework for medical image retrieval using machine learning and statistical similarity matching techniques with relevance feedback. IEEE Transact Inf Techn Biomed. 2007 Jan 2;11(1):58-69.

2. Morales M, Tapia L, Pearce R, Rodriguez S, Amato NM. A machine learning approach for feature-sensitive motion planning. In Algorithmic Foundations of Robotics VI 2004 Jul (pp. 361376). Springer, Berlin, Heidelberg.

3. Ireland G, Volpi M, Petropoulos GP. Examining the capability of supervised machine learning classifiers in extracting flooded areas from Landsat TM imagery: A case study from a Mediterranean flood. Remote sensing. 2015 Mar;7(3):3372-99.

4. Huang C, Davis LS, Townshend JR. An assessment of support vector machines for land cover classification. Int J Rem Sen. 2002 Jan 1;23(4):725-49.

5. Kotsiantis SB, Zaharakis I, Pintelas P. Supervised machine learning: A review of classification techniques. Emg Artif Intell Appl Comp Engg. 2007 Jun 10;160(1):3-24.

6. Cheriyadat AM. Unsupervised feature learning for aerial scene classification. IEEE Transactions on Geosci Rem Sen. 2013 Mar 7;52(1):439-51.

7. Bauer S, Nolte LP, Reyes M. Fully automatic segmentation of brain tumour images using support vector machine classification in combination with hierarchical conditional random field regularization. International conference on medical image computing and computer-assisted intervention 2011 Sep 18 (pp. 354361). Springer, Berlin, Heidelberg.

8. Liu K, Tong M, Xie S, Zeng Z. Fusing decision trees based on genetic programming for classification of microarray datasets. In International Conference on Intelligent Computing 2014 Aug 3 (pp. 126-134). Springer, Cham.

9. Dumitru D. Prediction of recurrent events in breast cancer using the Naive Bayesian classification. Annals of the University of Craiova-Mathematics and Computer Sci Series. 2009 Sep 11;36(2):92-6. 
10. Chou YH, Tiu CM, Hung GS, Wu SC, Chang TY, Chiang HK. Stepwise logistic regression analysis of tumour contour features for breast ultrasound diagnosis. Ultras Med Bio. 2001 Nov 1;27(11):1493-8.

11. Sarhan AM. Cancer classification based on microarray gene expression data using DCT and ANN. J Theor Appl Inf Techn. 2009 Aug 1;6(2).

12. Miah MB, Yousuf MA. Detection of lung cancer from CT image using image processing and neural network. In2015 International conference on electrical engineering and information communication technology (ICEEICT) 2015 May 21 (pp. 1-6).

13. Lu R, Marziliano P, Thng CH. Liver tumour volume estimation by semi-automatic segmentation method. In2005 IEEE Engineering in Medicine and Biology 27th Annual Conference 2006 Jan 17 (pp. 3296-3299).

14. Meng F, Li H, Liu G, Ngan KN. Image segmentation by incorporating colour reward strategy and active contour model. IEEE Trans Cybernt. 2013 Mar 7;43(2):725-37.

15. Corso JJ, Sharon E, Dube S, El-Saden S, Sinha U, Yuille A. Efficient multilevel brain tumour segmentation with integrated Bayesian model classification. IEEE Trans Med Imag. 2008 Apr 25;27(5):629-40.

16. Zhang K, Liu Q, Song H, Li X. A variational approach to simultaneous image segmentation and bias correction. IEEE Transact Cybernet. 2014 Oct 21;45(8):1426-37.

17. Miah MB, Yousuf MA. Detection of lung cancer from CT image using image processing and neural network. In2015 Inter- national conference on electrical engineering and information communication technology (ICEEICT) 2015 May 21;1-6.

18. Khobragade S, Tiwari A, Patil CY, Narke V. Automatic detection of major lung diseases using Chest Radiographs and classification by the feed-forward artificial neural network. In2016 IEEE 1st International Conference on Power Electronics, Intelligent Control and Energy Systems (ICPEICES) 2016 Jul 4;1-5. IEEE.

19. Tripathy RK, Mahanta S, Paul S. Artificial intelligence-based classification of breast cancer using cellular images. RSC Adv. 2014;4(18):9349-55.

20. D Deepa and Vignesh, R. and A, Sivasangari A and Mana, Suja Cherukullapurath and Samhitha, B. Keerthi and Jose, Jithina, Visualizing Road Damage by Monitoring System in Cloud (2020). Int J Electrical Engg Tech. 11(4), 2020, pp. 191-203.

21. Samhitha BK, Mana SC, Jose J, Vignesh R, Deepa D. Prediction of Lung Cancer Using Convolutional Neural Network (CNN). International Journal. 2020 May;9(3).

22. Vignesh R, Deepa D, Anitha P, Divya S, Roobini S. Dynamic enforcement of causal consistency for a Geo-replicated cloud storage system. Int J Elect Engg Techn. 2020;11(3).

23. Vignesh R, Deepa D, Mana SC, Samhitha BK. Secure K-NN Query on Encrypted Cloud Data with Multiple Keys. InJournal of Physics: Conference Series 2021 Mar 1;1770(1):012034. IOP Publishing. 